\title{
Combination of Independent Component Analysis and Feature Extraction of ERP for Level Classification of Sustained Attention
}

\author{
Farnaz Ghassemi, Student Member, IEEE, Mohammad Hasan Moradi, Member, IEEE, \\ Mahdi Tehrani Doust, and Vahid Abootalebi, Member, IEEE.
}

\begin{abstract}
This paper investigates the relations between ERP features and visual sustained attention. Continuous Performance Test is used for determining sustained attention level. Fifty eight features were extracted from the 19-channel recorded signals. Twenty four Subjects were divided into three classes according to their attention level. LDA classifier is used and high accuracy (94\%, $88 \%$ and $93 \%$ for each two classes) is achieved by using two features in classifying the test data. Obtained results are in agreement with the previous studies.
\end{abstract}

Keywords-Independent Component Analysis; Sustained Attention; Event Related Potential; LDA Classifier.

\section{INTRODUCTION}

Sustained attention refers to the ability to maintain a consistent behavioral response during continuous and repetitive processing of stimuli whose non-arousing qualities would otherwise lead to habituation and distraction to other stimuli $[1,2]$.

Evaluation of attention has various applications such as diagnosis and treatment of diseases (e.g. ADHD), learning enhancement in classroom, attention investigation in infants and attention monitoring in critical activities such as aeronautical navigation.

Unfortunately common methods for measuring attention evaluate its manifestations. Direct measurement of Attention using brain signals, is important because it can eliminate excessive interfaces. Benefits of achieving such a system are: minimizing individual effects such as responding speed or processing ability, application for subjects with vision/auditory disorders and independence of a specific language or culture.

Since Electroencephalography/ Event Related Potential (EEG/ERP) is the most direct noninvasive method for investigating brain activities and consequently attention, thus its evaluation is interesting for neuroscientists in sustained attention studies.

Up to now, different studies have been carried out

Manuscript received January 11, 2009.

F. Ghassemi is with the Biomedical Engineering Faculty of Amirkabir University of Technology, Tehran, Iran (corresponding author, phone: (+98)912-326-0661; fax: (+98)21-6642-0672; e-mail: ghassemi@aut.ac.ir).

M. H. Moradi is with the Biomedical Engineering Faculty of Amirkabir University of Technology, Tehran, Iran.

M. Tehrani Doust is with the Psychology Department, University of Tehran, Tehran, Iran, and Institute for Cognitive Science Studies (ICSS), Tehran, Iran. V. Abootalebi is with the Electrical Engineering Faculty of Yazd University, Yazd, Iran. regarding various types of attention and their relations with brain activities. Unfortunately, there are different states associated with sustained attention which are in broad use with varied ambiguous meanings such as arousal, alertness, vigilance and attention. This problem is investigated in [3] and it is mentioned that sustained attention can be used synonymously to vigilance and tonic alertness (not phasic alertness).

Makeig and coworkers investigated early and late ERPs during visual spatial attention and led to new grounds for attention extraction from brain signals by means of Independent Component Analysis (ICA) [4]. ICA is referred to the separation of independent sources which are mixed together with an unknown mixing matrix [5]. ICA method has been used in several studies $[4,6]$ to separate sources with brain origin from other sources with artifactual origins.

One of the most popular tests for evaluating sustained attention is Continuous Performance Test (CPT) [7]. Obtained results on EEG/ERP study during CPT [8] showed that all subjects shared a progressive backshift of alpha rhythm during the accomplishment of the CPT test while beta and gamma activities were stronger in the right hemisphere than in the left. An intense and widespread decrease in EEG spectral power during test performing became visible in many subjects. Statistical analysis revealed that EEG activity correlates with the test behavioral results in many cerebral areas.

Another study on a similar test for sustained attention has found two main ERP components (N2 and P3) investigating whether a central inhibitory mechanism intervenes to prevent the preparation and/or execution of a motor response. N2 was detectable in the No-Go trials. P3 presented a different scalp distribution based on the type of trial [9].

This paper investigates the correlations between sustained attention level and ERP morphological features especially P3 characteristics and then evaluates the validity and efficiency of these features to classify sustained attention levels.

\section{METHODS AND MATERIALS}

\section{A. CPT Task}

This study is based on the Conners' CPT II test which is a "No-Go" CPT task. The subjects seated on a comfortable chair with a place for relaxing the head. The test was performed in a quiet and dimly lit room. The distance between the subjects' eyes and monitor (19 inch) was $75 \pm 5 \mathrm{~cm}$ depending on their height. Different letters of English alphabet were presented randomly on the screen and subjects were required to click the left 
mouse button with the index finger of their dominant hand when any letter except the target "X" appeared. Subjects were instructed to respond as fast as they can, but also as accurately as possible.

The letters were $7.5 \mathrm{~cm}$ high and $7 \mathrm{~cm}$ wide which results in a $7^{\circ}$ visual angle. They appeared white colored on a black background. The inter-stimulus intervals (ISIs) were 1,2 or 4 seconds with a display time of $250 \mathrm{~ms}$. There were 6 blocks, with 3 sub-blocks each containing 20 trials. The order in which the different ISIs were presented varied between blocks. The experiment involved 360 stimuli: $36 \mathrm{X}$ letters (No-Go stimuli) and 324 other letters (Go stimuli). The test took approximately 14 minutes to complete.

\section{B. EEG Recordings}

19-channel EEG was recorded with $\mathrm{Ag} / \mathrm{AgCl}$ electrodes mounted in an electrode cap placed according to the international 10-20 standard (Fp1/Fp2, F3/F4, F7/F8, T3/T4, C3/C4, T5/T6, P3/P4, O1/O2, Fz/Cz/Pz). Average of A1 and A2 was used as reference. Three additional bipolar channels were used for vertical EOG recording, synchronization of CPT system with EEG signals and recording of subject's responses. For the last two pairs of channels, two independent isolator circuits were used for providing the subjects' safety and reducing noise. A 32-channel AC/DC amplifier (Walter) was used for data recording and Pl-Winsor 3.0 for data acquisition. Amplifier band pass was $0.05-100 \mathrm{~Hz}$ and a $50 \mathrm{~Hz}$ notch filter was used for line noise reduction. The A/D sampling rate was $200 \mathrm{~Hz}$. Impedance of all electrodes was kept below $5 \mathrm{~K} \Omega$.

\section{Subjects}

Twenty four volunteers (14 male) with average age of $29 \pm 6.3$ years participated in the experiment. Since financial incentives affect the sustained attention level [3], subjects were not paid to participate and they were just thanked with a small gift at the end of the experiment. All subjects were tested for handiness with Edinburgh test (1 left-handed). They had normal or corrected to normal vision. For evaluating generalization (as the test is in W/B), all subjects were checked for colorblindness with Ishihara test and one male was color-blind. For evaluating subject's Neuropsychological history SARs test was used and subjects were free of neurological disorders and were not taking any medication. All subjects gave their informed consent. The experiment was conducted in accordance with the Declaration of Helsinki.

\section{ERP Extraction}

Signals were filtered by a $0.1-85 \mathrm{~Hz}$ band pass filter. Combination of Efficient Variant of Fast ICA (EFICA) and ${ }^{1}$ Efficient Weights Adjusted SOBI (EWASOBI) was used as the ICA method [10]. Time, frequency and spatial characteristics of the resulted independent components were studied and proper components with brain origins which were physiologically plausible were selected manually (from 1 to 6 components in different subjects or ISIs). Then only these components were returned to electrode space.

Four groups were considered for each subject: Target stimuli (X), Non-target stimuli (nX), Correct answered target stimuli (CX, which is the $\mathrm{X}$ that subject did not respond) and Non-correct answered target stimuli (nCX).
Twelve ERPs were extracted for each subject for different ISIs $(1,2$ and $4 \mathrm{~s})$ and groups $(\mathrm{X}, \mathrm{nX}, \mathrm{CX}, \mathrm{nCX})$. Period of epochs were considered $200 \mathrm{~ms}$ before till $1000 \mathrm{~ms}$ after stimuli onset and ERPs were achieved by averaging epochs time-locked to stimuli onset.

The P3 window was considered at $350-650 \mathrm{~ms}$ after stimulus onset based on the prior researches [9, 11]. Amplitude of P3 is defined as the difference between the mean prestimulus baseline voltage and the largest positive-going peak of the ERP waveform within a time window (e.g. 350$650 \mathrm{~ms}$ )[11]. Amplitude and latency of P3 were both computed.

\section{E. Features and Classifier}

Defined features included two general groups of amplitude and delay of P3 which were calculated for different ISIs and groups. In addition, features were defined for absolute ISIs or relative to each other. Therefore 48 features $(2 * 3 * 4 * 2)$ were considered for each channel.

Subjects divided to three classes according to their attention level, 7 in high (H), 7 in medium (M) and 10 in low (L) attention class. For investigating the relation between these features and CPT results, Pearson correlation was calculated between attention levels and features for different channels. Fifty eight features had significant correlation $(\mathrm{P}$-value $<0.05)$ with attention levels which are expressed in Table. 1. Symbol "A" is used for amplitude and "D" for delay of P3. The phrase in the parenthesis explains the group and subscript indices express the related ISI of calculated ERP which could be absolute or relative. For example, $\mathrm{D}_{\mathrm{S} 1}(\mathrm{CX})$ means the delay of $\mathrm{P} 3$ on ERP calculated for $1 \mathrm{~s}$ ISI in correct $\mathrm{X}$ group or $\mathrm{A}_{\mathrm{S} 24}(\mathrm{nCX})$ means the difference of amplitudes of P3 on ERP

Table 1. Feature Characteristics.

Features which had significant correlation with sustained attention level are defined. For example $\mathrm{A}_{524}(\mathrm{nCX})$ means the difference of amplitudes of $\mathrm{P} 3$ on ERP calculated for $2 \mathrm{~s}$ and $4 \mathrm{~s}$ intervals in noncorrect $\mathrm{X}$ group. The left number under each feature expresses its correlation coefficients with sustained attention level while the right number is that features number.

\begin{tabular}{|c|c|c|c|c|c|}
\hline \multirow{2}{*}{ 弌 } & \multicolumn{5}{|c|}{ Feature } \\
\hline & \multicolumn{5}{|c|}{ Correlation /Feature No } \\
\hline $\mathbf{F} \mathbf{p}_{1}$ & 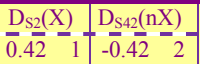 & & $\mathbf{F} \mathbf{p}_{2}$ & 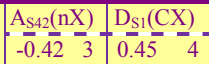 & $=\frac{\mathrm{D}_{\mathrm{S} 2}(\mathrm{CX})}{0.47}-{ }_{5}$ \\
\hline $\mathbf{F}_{7}$ & 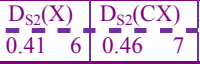 & $\left\{\begin{array}{l}\mathrm{D}_{\mathrm{S} 4}(\mathrm{nCX}) \\
-0.41\end{array}\right.$ & $\mathbf{F}_{8}$ & 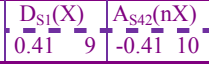 & 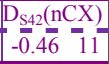 \\
\hline $\mathbf{F}_{3}$ & 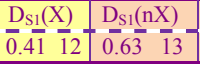 & $\left\{\begin{array}{l}\mathrm{A}_{\mathrm{S} 1}(\mathrm{CX}) \\
-0.52 \\
-0.54\end{array}\right.$ & $\begin{array}{l}\mathrm{D}_{\mathrm{S} 1}(\mathrm{CX}) \\
0 . \overline{43} \\
15\end{array}$ & & \\
\hline $\mathbf{F}_{4}$ & 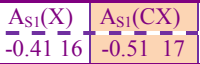 & $\left\{\begin{array}{l}\mathrm{D}_{\mathrm{S} 1}(\mathrm{CX}) \\
\overline{0.44}-1 \overline{8}\end{array}\right.$ & & & \\
\hline $\mathbf{T}_{3}$ & $\begin{array}{l}\mathrm{D}_{\mathrm{S} 1}(\mathrm{nX}) \\
0.44 \\
0.49\end{array}$ & & $\mathbf{T}_{4}$ & \begin{tabular}{|cc|cc|}
$\mathrm{D}_{\mathrm{S} 1}(\mathrm{X})$ & $\mathrm{D}_{\mathrm{S1}}(\mathrm{CX})$ \\
0.49 & 20 & 0.46 & 21
\end{tabular} & \\
\hline $\mathrm{C}_{3}$ & 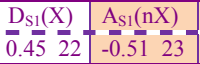 & $\left\{\begin{array}{l}\mathrm{D}_{\mathrm{S1}}(\mathrm{nX}) \\
0.56 \quad 24\end{array}\right.$ & $\frac{\mathrm{D}_{\mathrm{S} 1}(\mathrm{CX})}{0.5125}$ & & \\
\hline $\mathrm{C}_{4}$ & 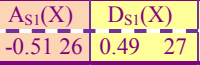 & $\frac{\mathrm{D}_{\mathrm{S} 2}(\mathrm{X})}{0.4 \overline{2}}-$ & $\mathrm{D}_{\mathrm{S} 4}(\mathrm{X})$ & $\begin{array}{llll}\mathrm{D}_{\mathrm{S} 2}(\mathrm{nX}) & \mathrm{A}_{\mathrm{S} 1}(\mathrm{CX}) \\
0.45 & \overline{3} 0 & -0.6 \overline{8} & 31 \\
\end{array}$ & $=\frac{\mathrm{D}_{\mathrm{S} 1}(\mathrm{CX})}{0.50} \overline{32}$ \\
\hline T5 & 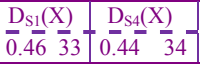 & $\frac{\mathrm{D}_{\mathrm{S} 1}(\mathrm{CX})}{0.4 \overline{8}}-\frac{}{35}$ & T6 & $\frac{\mathrm{D}_{\mathrm{S} 41}(\mathrm{X})}{0.42}$ & \\
\hline $\mathbf{P}_{3}$ & 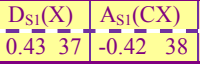 & $\left\{\begin{array}{l}\mathrm{D}_{\mathrm{S1}}(\mathrm{CX}) \\
0.56 \\
3.59\end{array}\right.$ & $\mathbf{P}_{4}$ & 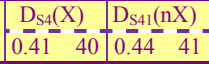 & \\
\hline $\mathbf{O}_{1}$ & \begin{tabular}{ll|ll}
$\mathrm{D}_{\mathrm{S} 42}(\mathrm{X})$ & $\mathrm{D}_{\mathrm{S} 41}(\mathrm{nCX})$ \\
0.46 & $\overline{4}$ & 0.45 & $\overline{4}$ \\
\end{tabular} & $\left\{\begin{array}{l}\mathrm{A}_{\mathrm{S} 42}(\mathrm{nCX}) \\
0.42 \\
0.4 \frac{4}{4}\end{array}\right.$ & $\mathrm{O}_{2}$ & \begin{tabular}{|lllll}
$\mathrm{D}_{\mathrm{S} 42}(\mathrm{X})$ & $\mathrm{D}_{\mathrm{S} 42}(\mathrm{nX})$ \\
$0.4 \overline{3}$ & $\overline{45}$ & $-0.4 \overline{5}$ & $\overline{4} 6$ \\
\end{tabular} & \\
\hline $\mathbf{F}_{\mathbf{Z}}$ & 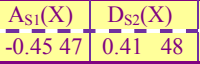 & $\left\{\begin{array}{l}\mathrm{D}_{\mathrm{S1}}(\mathrm{nX}) \\
0.4 \overline{9}-4 \overline{9}\end{array}\right.$ & $\frac{\mathrm{A}_{\mathrm{S} 1}(\mathrm{CX})}{-0.61}$ & 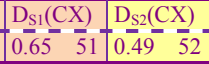 & \\
\hline $\mathbf{C}_{\mathbf{Z}}$ & 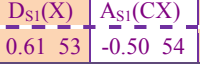 & $\frac{\mathrm{D}_{\mathrm{S} 1}(\mathrm{CX})}{0.60}-$ & $\mathbf{P}_{\mathbf{Z}}$ & $\begin{array}{cccc}\mathrm{D}_{\mathrm{S} 41}(\mathrm{nX}) & \mathrm{D}_{\mathrm{S} 42}(\mathrm{nX}) \\
0.55 & 56 & 0.45 & -57\end{array}$. & $=$ \\
\hline
\end{tabular}


calculated for $2 \mathrm{~s}$ and $4 \mathrm{~s}$ ISIs in non-correct $\mathrm{X}$ group.

In the next step, Linear Discriminant Analysis (LDA) classifier was used for discrimination of each two classes. According to Leave One Out (LOO) method, one subject's data was considered as test data and the classifier was trained based on others, then it was evaluated on the test data. This procedure was repeated for all subjects and accuracy is achieved by the ratio of correct classifications to total number of subjects. This test was performed for each feature and then repeated for all combinations of two features.

\section{RESULTS}

Grand average ERP for all subjects is illustrated in fig. 1. As it can be seen, amplitude of P3 is obviously larger in target (X) than non-target $(\mathrm{nX})$ stimuli. These curves are smoothed with a $12 \mathrm{~Hz}$ filter. Fig. 2 demonstrates the topographic map of ERP at different delays (columns) for 1s ISI in correct $\mathrm{X}$ stimuli. Each row contributes to one attention level. It can be seen that there are some outstanding differences between classes. These maps are obtained using EEGLAB software [12].

Calculated correlations between CPT results and defined features showed a significant relation $(\mathrm{P}$-value $<0.05)$ at all locations of the scalp (Table 1).Accuracy for classification on test and train data with one and two features are shown in Table 2 . The best result for classification between $\mathrm{H} / \mathrm{L}$ class on test data $(94 \%)$ is obtained with combination of features 43 and 52 which are related to $\mathrm{D}_{\mathrm{S} 41}(\mathrm{nCX})$ and $\mathrm{D}_{\mathrm{S} 2}(\mathrm{CX})$ according to table 1 . The best result for classification between $\mathrm{H} / \mathrm{M}$ class on test data (88\%) is obtained with combination of features 43 and 51 which are related to $\mathrm{D}_{\mathrm{S} 41}(\mathrm{nCX})$ and $\mathrm{D}_{\mathrm{S} 1}(\mathrm{CX})$ while the best result for classification between $\mathrm{M} / \mathrm{L}$ class on test data $(93 \%)$ is obtained with combination of features 11 and 58 which are related to $\mathrm{D}_{\mathrm{S} 42}(\mathrm{nCX})$ and $\mathrm{D}_{\mathrm{S} 1}(\mathrm{CX})$. The accuracy for classification of all classes is $79 \%$ which obtained by combination of features 43 and $23\left(\mathrm{D}_{\mathrm{S} 42}(\mathrm{nCX})\right.$ and $\left.\mathrm{A}_{\mathrm{S} 1}(\mathrm{nX})\right)$. These percents are summarized in fig. 3 .

\section{DISSCUSION}

The P3 peak on target stimuli (X) was clearly observed for all subjects (irrespective of answers correctness) and this P3
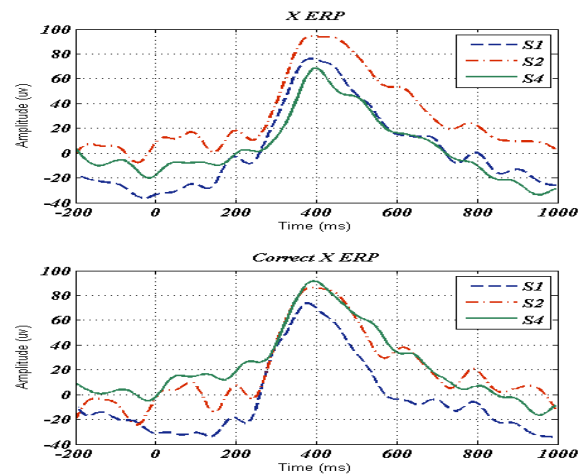

observation on unusual target stimuli is in complete agreement with previous studies $[9,11,13]$. This peak was obviously larger on target than non-target stimuli as can be seen in fig.1. This evident could be due to characteristics of task, complete perception of task for subjects and/or the proper ratio of target stimuli to total stimuli (1 to 10).

Independent components for EEG signals were calculated and best components were selected based on their time, frequency and spatial characteristics. Calculated correlations confirm the significant relations between CPT result and some ERP features at all scalp locations but it was stronger at $\mathrm{C} 4$, $\mathrm{C} 3, \mathrm{Cz}, \mathrm{F} 3, \mathrm{~F} 4, \mathrm{Fz}, \mathrm{P} 3$ and Pz. Best results of classification in all classes $(79 \%)$ was obtained with combination of two features, one amplitude and one delay $\left(\mathrm{D}_{\mathrm{S} 42}(\mathrm{nCX})\right.$ and $\left.\mathrm{A}_{\mathrm{S} 1}(\mathrm{nX})\right)$, while in classification of each two classes $(\mathrm{H} / \mathrm{L} 94 \%$, $\mathrm{H} / \mathrm{M} 88 \%, \mathrm{M} / \mathrm{L} 93 \%$ ), these two features were both for delay but one on CX group and the other on $\mathrm{nCX}$ group $\left(\mathrm{D}_{\mathrm{S} 41}(\mathrm{nCX})\right.$ $\mathrm{D}_{\mathrm{S} 2}(\mathrm{CX}), \quad \mathrm{D}_{\mathrm{S} 41}(\mathrm{nCX})-\mathrm{D}_{\mathrm{S} 1}(\mathrm{CX}), \quad$ and $\quad \mathrm{D}_{\mathrm{S} 42}(\mathrm{nCX})-\mathrm{D}_{\mathrm{S} 1}(\mathrm{CX})$ respectively). Consequently results represent a significant relation between CPT result and some parameters of brain signals which can be used in evaluating the level of attention.

\section{ACKNOWLEDGMENT}

We thank "Institute for Cognitive Science Studies" (ICSS) for using their EEG laboratory for performing the tests.

\section{REFERENCES}

[1] E. Kandel, J. Schwartz, \& T. Jessell, Visual Attention. "Principles of Neural Science." $4^{\text {th }}$ ed. Vol. 1: Mc Grow Hill, 2000.

[2] I. H. Robertson, T. Manly, J. Andrade, B. T. Baddeley, \& J. Yiend, "Oops!: Performance correlates of everyday Attentional failures in traumatic brain injured and normal subjects." Neuropsychologia, 24(5), pp. 636-647, 1997.

[3] B.S. Oken, M.C. Salinsky, \& S.M. Elsas, "Vigilance, alertness, or sustained attention:physiological basis and measurement." Clinical Neurophysiology, 117, pp.1885-1901, 2006.

[4] S. Makeig, M. Westerfield, J. Townsend, T-P. Jung, E. Courchesne, \& T. J. Sejnowski, "Functionally independent components of the early event-related potential in a visual spatial attention task." Philosophical Transactions of the Royal Society: Biological, 1999. Vols. 354 1135-44.

[5] Hyvarinen A., Karhunen J., Oja E., "Independent Component AnalysisTheory and Applications," John Wiely \& Sons, 2001.
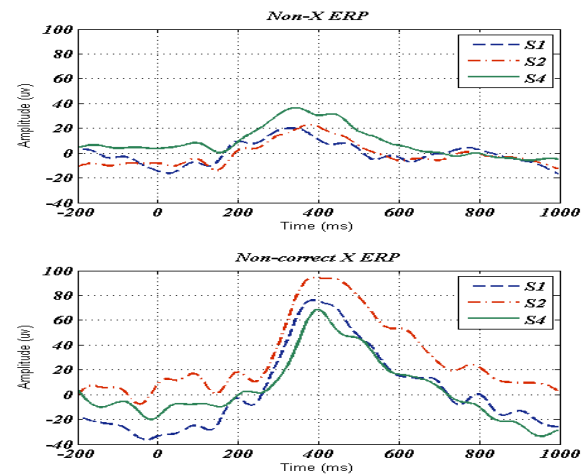

Figure 1. Grand average ERP for all subjects.

ERPs for $1 \mathrm{~s}$ interval are illustrated with dash blue line, ERPs for $2 \mathrm{~s}$ interval are illustrated with dash-dot red line and ERPs for $4 \mathrm{~s}$ interval are illustrated with solid green line. Four different groups of ERP $(\mathrm{X}, \mathrm{nX}, \mathrm{CX}, \mathrm{nCX})$ are demonstrated in distinct subplots. Amplitude of P3 is obviously larger in target (X) than non-target (nX) stimuli. 


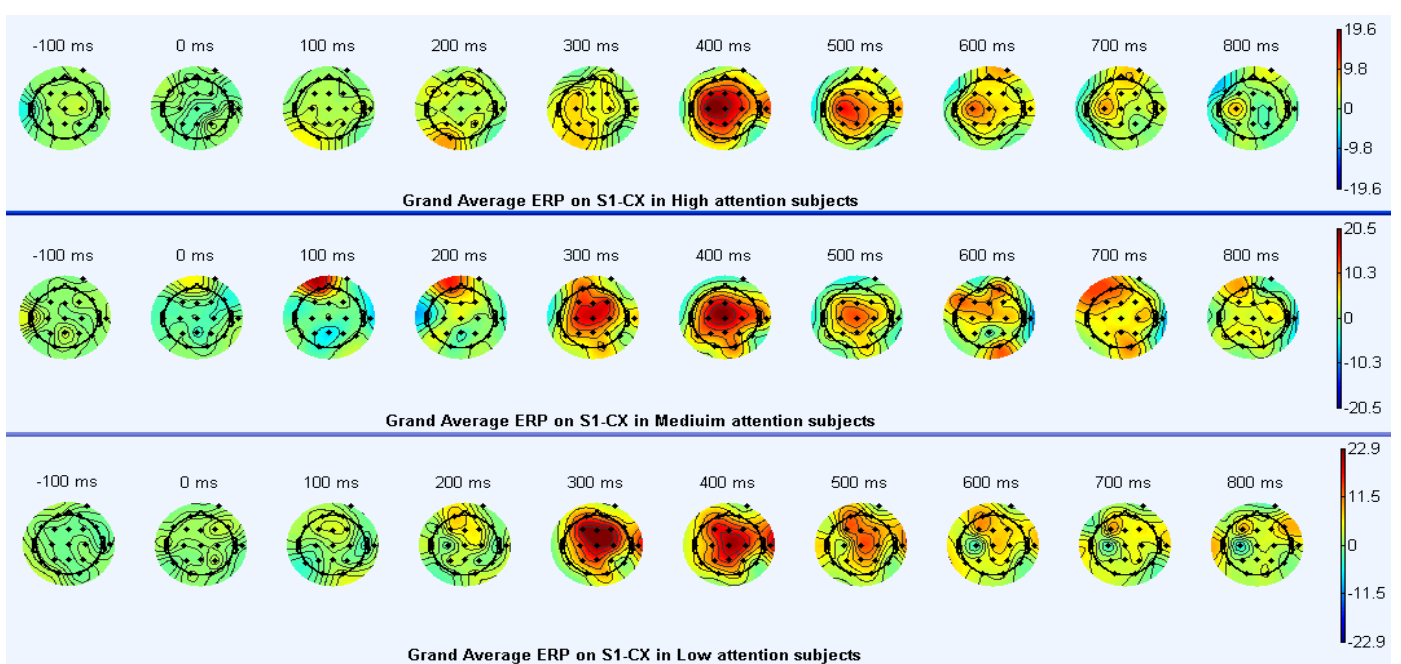

Table 2. The accuracy achieved with LDA classifier.

The accuracy is calculated for test and train data for classification of each two classes with 1 feature (first three lines) and 2 features (last three lines). Last column is the average of all classifications ( 3 cases) for each feature or combination of features.

Figure 2. Topographic maps for 1s ISI in correct $\mathrm{X}$ stimuli at different time delays according to target presentastion.

First row demonstrates the grand average ERP in High attention subjects and the grand average ERP in Medium and Low attention subjects are plotted in middle and down rows consequently. Maximum amplitude (marked on color bar of each plot) and delay of peak are different amog classes.

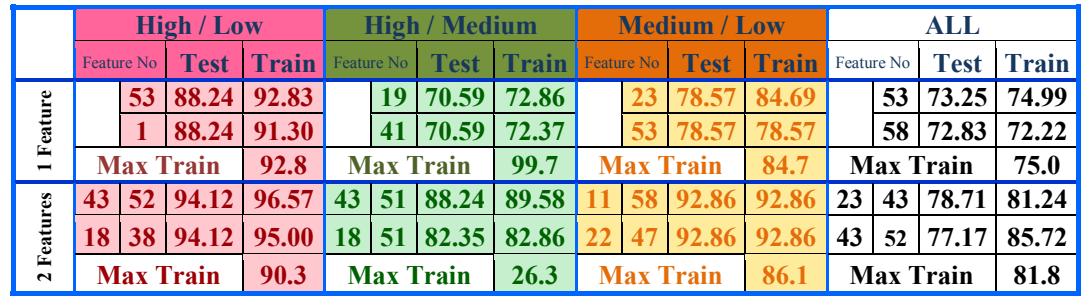

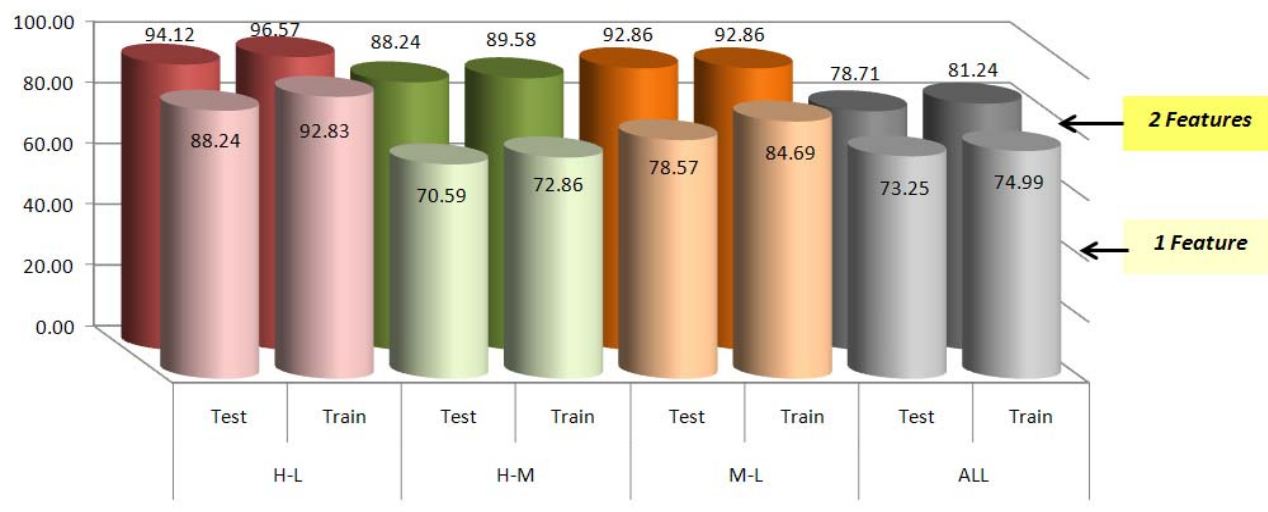

Figure 3. Chart of achieved accuracy.

The front row shows the accuracies achieved with one feature and the back row demonstrates accuracies achieved with two features.

[6] J. Onton, S. Makeig, "Information-Based Modeling of Event-Related Brain Dynamics, " Neuper \& Klimesch (Eds.), Progress in Brain Research, Vol. 159, Ch. 7, 2006.

[7] H. E. Rosvold, A. F. Mirsky, I. Sarason, E. D. Jr. Bransome, \& L. K. Beck, "A continuous performance test of brain damage." Journal of Consulting Psychology, 20, 343-350, 1956.

[8] E. Molteni, A. M. Bianchi, M. Butti, G. Reni, \& C. Zucca, "Analysis of the dynamical behaviour of the EEG rhythms during a test of sustained attention," Proceedings of the 29th Annual International Conference of the IEEE EMBS Cité Internationale, Lyon, France August 23-26, 2007.
[9] L. Zordan, M. Sarlo, \& F. Stablum, "ERP components activated by the "GO!" and "WITHHOLD!" conflict in the random Sustained Attention to Response Task." Brain and Cognition, 66, pp. 57-64, 2008.

[10] http://read.pudn.com/downloads86/sourcecode/math/333232/combi.m .htm

[11] J. Polich, "Updating P300: An integrative theory of P3a and P3b," Clinical Neurophysiology, 118, pp.2128-2148, 2007.

[12] http://www.sccn.ucsd.edu/eeglab/

[13] V. Abootalebi, M. Moradi, M. Khalilzadeh, A comparison of methods for ERP assessment in a P300-based GKT, International Journal of Psychophysiology, 62, pp.309-320, 2006. 